\title{
The new spin physics program of the COMPASS experiment
}

\author{
Luís Silva ${ }^{1, a, b}$ \\ ${ }^{1}$ LIP - Laboratório de Instrumentação e Física Experimental de Partículas, Av. Elias Garcia 14, 1ํ 1000 \\ Lisboa, Portugal.
}

\begin{abstract}
The COMPASS experiment, at CERN SPS, has been compiling for more than a decade successful and precise results on nucleon structure and hadron spectroscopy, leading to statistical errors much smaller than previously measured. The new COMPASS spin physics program, starting this year, aims to a rather complete nucleon structure description; this new representation goes beyond the collinear approximation by including the quark intrinsic transverse momentum distributions. The theoretical framework, for this new picture of the nucleon, is given by the Transverse Momentum Dependent distributions (TMDs) and by the Generalised Parton Distributions (GPDs). The TMDs, in particular Sivers, Boer-Mulders, pretzelosity and transversity functions will be obtained through the polarised Drell-Yan process, for the first time. The results will be complementary to those already obtained via polarised Semi-Inclusive Deep Inelastic Scattering (SIDIS). Also unpolarised SIDIS will be studied, allowing the knowledge improvement of the strange quark PDF and the access to the kaon fragmentation functions (FFs). Deeply Virtual Compton Scattering (DVCS) off an unpolarised hydrogen target will be used to study the GPDs, in a kinematic region not yet covered by any existing experiment.
\end{abstract}

\section{Introduction}

The fixed target experiment COMPASS (COmmon Muon and Proton Apparatus for Structure and Spectroscopy) is located in the Super Proton Synchrotron (SPS) facility, at CERN. The experiment started in 2002 and since then regularly takes data every year, having had only two interruptions for upgrades (on 2005 and 2013). COMPASS uses polarised muons and hadrons on protons and deuterons. The experimental apparatus is a fifty meter long two-stage spectrometer, followed by a cryogenic polarised target system. The first spectrometer stage is designed for low momentum particles having relatively large angles. The second spectrometer is focused to measure high momentum particles having small angles. More information about the COMPASS spectrometer can be found in Ref. [1].

The previous COMPASS program can be divided into two physics sub-programs: one, so-called muon program, for nucleon structure studies and another, so-called hadron program, for hadron spectroscopy investigations. In the muon program, the beam is composed by naturally and longitudinally polarised positive muons (at energies of 160 and $200 \mathrm{GeV} / \mathrm{c}$ ), which scatter off protons and deuterons,

\footnotetext{
a e-mail: 1silva@lip.pt

${ }^{\mathrm{b}}$ On behalf of the COMPASS Collaboration.
} 
both longitudinal and transverse polarised. For the hadron program, unpolarised hadrons -pions, kaons and protons- (at energies of 160 and $190 \mathrm{GeV} / \mathrm{c}$ ) scatter off unpolarised protons and deuterons.

After a decade of precise and successful results, the COMPASS collaboration is performing a new spin physics program [2], approved in December 2010 and started in 2012. The new program aims to a more complete knowledge of the nucleon structure, going beyond the collinear approximation by taking into account the quark intrinsic transverse momentum distributions. This new approach allows to introduce a new theoretical frame: the Generalised Parton Distributions (GPDs), and in the same direction nevertheless with a different formulation, the Transverse Momentum Dependent Distributions (TMDs). Also two other subjects will be addressed, the unpolarised PDFs and TMD effects in Semi-Inclusive Deeply Inelastic Scattering (SIDIS).

\section{Unpolarise Semi-Inclusive Deep Inelastic Scattering}

Semi-Inclusive Deep Inelastic Scattering is the standard tool used to investigate the inner structure of the nucleon and therefore to probe the nucleon contents. In figure 1. this process is illustrated by a schematic representation; a muon particle scatters off a nucleon, the interaction is done electromagnetically via a virtual photon with one of the valence quarks, the nucleon absorbing the imparted energy by the photon breaks up producing the final state that afterwards fragments into hadrons. The purpose of SIDIS is to study these hadrons.

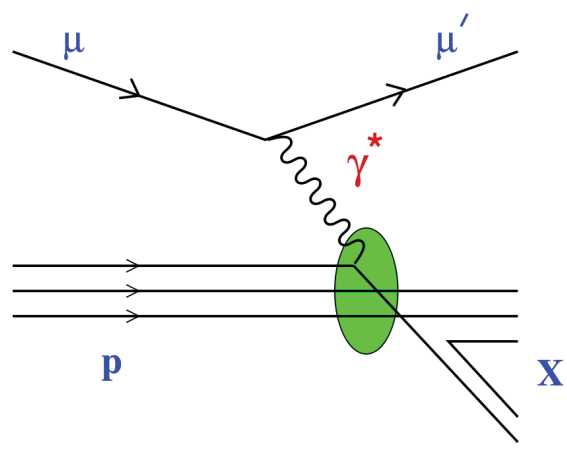

Figure 1: Schematic representation of the Semi-Inclusive Deep Inelastic Scattering.

The muon program of COMPASS was mostly focused on polarised Semi-Inclusive Deep Inelastic Scattering (SIDIS) using a ${ }^{6} \mathrm{LiD}$ (deuteron) target, some of the latest results can be found on these references [3-5]. In this new program, the purpose of the unpolarised SIDIS data is to increase the existing statistics. This time a liquid hydrogen target will be used to avoid nuclear effects. These new data together with existing COMPASS SIDIS data on the deuteron will allow quark flavour separation, in particular to measure several hadron multiplicities: $\pi^{ \pm}, \pi^{0}, K^{ \pm}, K^{0}, \Lambda$ and $\bar{\Lambda}$. The new proton data will be obtained in a region of $\mathrm{x}$-Bjorken (thereafter denoted by $x$ ), where measurements from other experiments are either not available or have limited precision. These new data will be also used in QCD global analyses to constraint the Parton Distribution Functions (PDFs) and also for the fragmentation functions. In particular for the strange quark, COMPASS can give a valuable contribution to constrain the quark distribution $s(x)$ in the region of high $x(0.004<x<0.6)$. Figure 2 (a). illustrates the impact of the COMPASS contribution to the strange quark distribution, $s(x)$, where the parametrisations of two global analyses are shown, also in the $x$-axis the range that COMPASS can 
constrain is depicted. In figure 2 (b). the $s(x)+\bar{s}(x)$ distribution determined by the HERMES collaboration [6] using SIDIS kaon data on deuteron is depicted, this result disagrees substantially with the existing parametrisations. Recently, HERMES published a new re-evaluation of the strange quark distribution [7], which has is more precise, yet still in disagreement with the theoretical parametrisations. Also in figure 2 (b). the COMPASS projection of statistical error for one week of data taking with the liquid hydrogen target is shown.

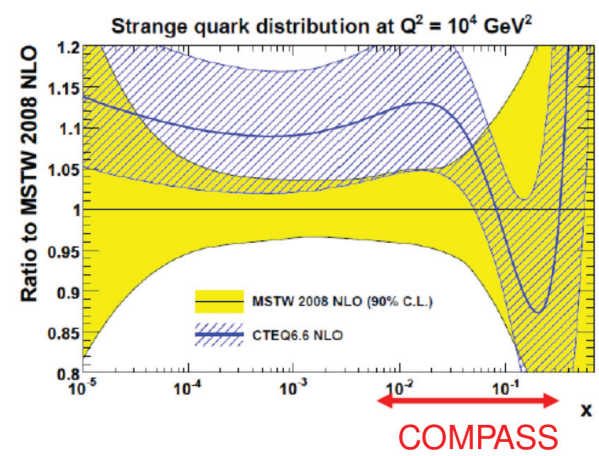

(a)

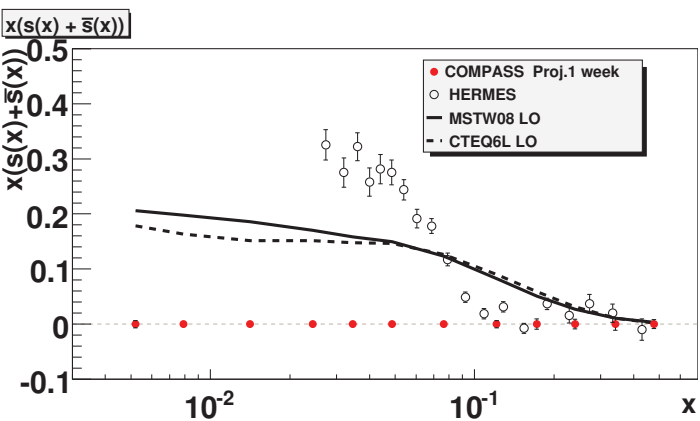

(b)

Figure 2: (a) Strange quark distribution, at $Q^{2}=10^{4} \mathrm{GeV}^{2}$, obtained using QCD global analysis from CTEQ and MSTW groups [8]. On the $x$-axis the range of COMPASS contribution is shown. (b) Projected statistical error for $s(x)+\bar{s}(x)$ for one week of data taking with the liquid hydrogen target, also shown are the parametrisations of CTEQ and MSTW groups and the HERMES result [6].

\section{Generalised Parton Distribution Functions}

The Generalised Parton Distribution Functions (GPDs) are a conjugation of both nucleon electromagnetic form factors and PDFs. GPDs provide transverse spatial information of a parton as a function of the fraction it carries of the nucleon's longitudinal momentum, i.e. they correlate (transverse) spatial and (longitudinal) momentum degrees of freedom of quarks and gluons. The study of the GPDs can be performed experimentally by the Deeply Virtual Compton Scattering (DVCS) and Deeply Vector Meson Production (DVMP) mechanisms.

There are four GPDs: $H^{f}, E^{f}, \widetilde{H}^{f}$ and $\widetilde{E}^{f}$, where $f$ can be one of the quarks $u, d$ or $s$ and gluon $g$. The GPDs attracted special attention after it was shown that the total angula momentum os a given parton species $f$ is related to the $2^{\text {nd }}$ moment of the sum of the two GPDs $H^{f}$ and $E^{f}$, by the Ji relation [9]:

$$
J^{f}\left(Q^{2}\right)=\frac{1}{2} \lim _{t \rightarrow 0} \int_{-1}^{1} \mathrm{~d} x^{\prime} x^{\prime}\left[H^{f}\left(x^{\prime}, \xi, t, Q^{2}\right)+E^{f}\left(x^{\prime}, \xi, t, Q^{2}\right)\right],
$$

where $x^{\prime}$ is an internal variable that is integrated over in a convolution of the given GPD with a kernel describing the hard virtual-photon quark interaction, $Q^{2}$ is the photon virtuality, $t$ is the total four-momentum squared transferred between the initial and final state and $\xi=x /(2-x)$, where $x$ is $\mathrm{x}$ Bjorken variable. Equation (1) provoked quite a lot of experimental and theoretical activities towards determinations of GPDs; at the same time it opened the new way to constrain the contributions $J^{q}$ 
from total quark angular momenta to the nucleon's spin budget

$$
\frac{1}{2}=\sum_{q=u, d, s} J^{q}\left(Q^{2}\right)+J^{g}\left(Q^{2}\right),
$$

where $J^{g}$ is the total gluon contribution to the nucleon spin. The Ji relation (1) can be also a way to constrain the quark total angular momentum contributions to the nucleon spin budget.

A particular simplification of the GPDs as probability density is done in the limiting case $\xi=0$ where the parton carries the same longitudinal momentum fraction $x^{\prime}$ in initial and final state and hence the momentum transfer $t \equiv-\Delta^{2}=-\Delta_{L}^{2}-\Delta_{\perp}^{2}$ is purely transverse, $t=-\Delta_{\perp}^{2}$. In this case the Fourier transform of the $-\Delta_{\perp}^{2}$ dependence of the GPD $H^{f}\left(x, 0,-\Delta_{\perp}^{2}\right)$ for fixed $x$ describes the spatial distribution of partons of species $f$ carrying the longitudinal momentum fraction $x^{\prime}$, with respect to their transverse distance $b_{\perp}$ from the centre of momentum of the nucleon (impact-parameter representation) [10]

$$
q^{f}\left(x, b_{\perp}\right)=\int \frac{\mathrm{d}^{2} \Delta_{\perp}}{(2 \pi)^{2}} e^{-i \Delta_{\perp} \cdot b_{\perp}} H^{f}\left(x, 0,-\Delta_{\perp}^{2}\right)
$$

The 3-D impact-parameter-dependent parton distribution $q^{f}\left(x, \Delta_{\perp}\right)$ can be interpreted as providing a set of "tomographic images" of the nucleon, as illustrated by the cartoon shown in figure 3 . where depending on the slice of $x$ different regions of the nucleon can be accessed.

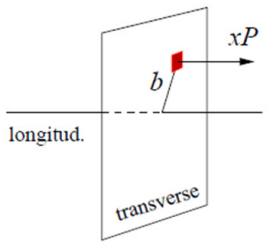

(a)

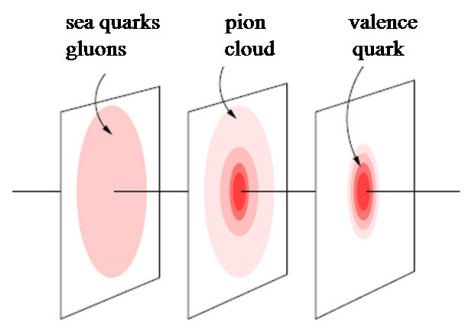

(b) $x \sim 0.003 \quad x \sim 0.03 \quad x \sim 0.3$

Figure 3: Nucleon tomography: (a) The Fourier transform of the $-\Delta_{\perp}^{2}$ dependence of the GPD $H^{f}\left(x, 0,-\Delta_{\perp}^{2}\right)$ for fixed $x$ describes the distribution of the transverse distance $b \equiv\left|b_{T}\right|$ of partons carrying the fraction $x$ of the nucleon's longitudinal momentum $P$, from the centre of momentum of the nucleon. (b) Sketch of tomographic views of the transverse spatial parton distribution in the nucleon at certain parton longitudinal momentum fractions $x$.

Another interesting result comes from $x^{\prime}=\xi$. It has no probabilistic interpretation but nevertheless its Fourier transform is connected to the distance $r_{\perp}$ between struck parton and spectator system $[11,12]$. At leading order in $\alpha_{s}$, the corresponding average $\left\langle r_{\perp}^{2}(x)\right\rangle$ can be directly obtained from the imaginary part of the amplitudes of exclusive processes. At small $x$, where amplitudes are predominantly imaginary, one has the relation $\left\langle r_{\perp}^{2}\left(x_{B}\right)\right\rangle \approx 2 \cdot B(x)$ if the exclusive cross section is parametrised as $\frac{\mathrm{d} \sigma}{\mathrm{d} t} \propto \exp (-B(x)|t|)$. Figure 4. shows the projected statistical and systematic uncertainties for a measurement of the $x_{B}$ dependence of the so-called $t$-slope parameter $B(x)$ for the range $1 \leq Q^{2} \leq 8 \mathrm{GeV}^{2} . B(x)$ constitutes a direct measurement of the DVCS cross section. 


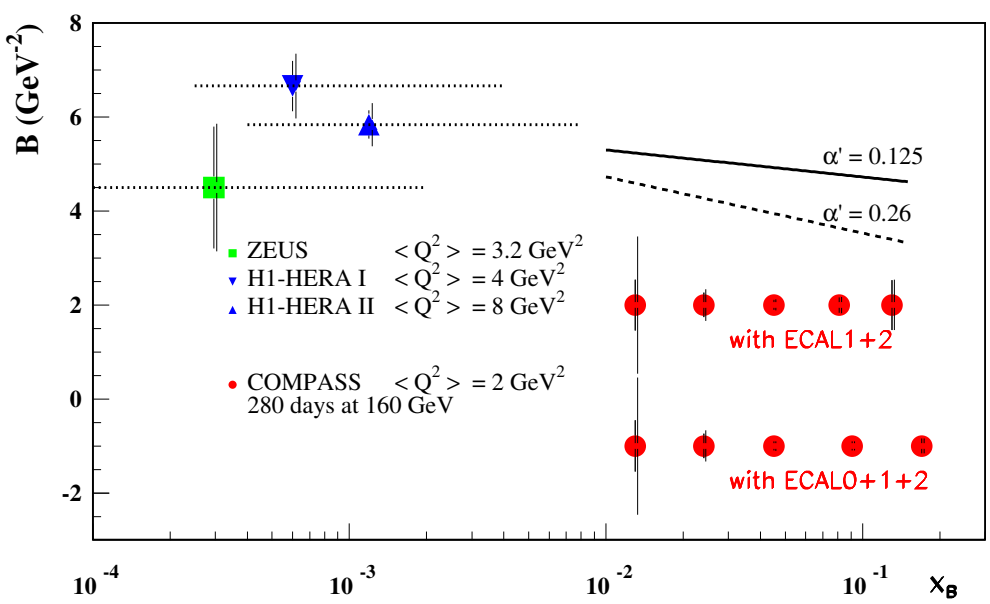

Figure 4: Projections for the $x$ dependence of the $t$-slope parameter $B(x)$ of the DVCS cross section, calculated for $1<Q^{2}<8 \mathrm{GeV}^{2}$. For comparison some HERA results with similar $\left\langle Q^{2}\right\rangle$ are shown [13-15], for which the horizontal dashed lines indicate their $x$ range. The left vertical bar on each data point indicates the statistical error only while the right one includes also the quadratically added systematic uncertainty, using only ECAL1 and ECAL2 (first row) and also ECAL0 (second row). Two different parametrisations are shown using $\alpha^{\prime}=0.125 \mathrm{GeV}^{-2}$ and $0.26 \mathrm{GeV}^{-2}$.

\section{Transverse Momentum Dependent Distributions}

In the so-called leading twist regime, where the transverse intrinsic momentum distributions of the partons, $k_{T}$, is neglected, the nucleon structure is described by three PDFs, namely: (1) the unpolarised distribution function $f_{1}(x)$, also known as the number density, describing the probability of finding a quark with fraction $x$ of longitudinal momentum of the parent nucleon, regardless its spin orientation, (2) the helicity distribution $g_{1}(x)$, representing the difference between the number densities of quarks with spin parallel and anti-parallel to the spin of the longitudinally polarised parent nucleon, and (3) the transversity function $h_{1}(x)$, analogous to $g_{1}(x)$ but for the transverse orientation of the quark spins and nucleon polarisation. Several experimental observations on large azimuthal and spin asymmetries are not be explained in perturbative QCD at leading twist. The new approach are the Transverse Momentum dependent parton Distribution functions (or simply TMDs), which aim to describe the nucleon structure by including the transverse intrinsic momentum distributions of the partons. In the case where $k_{T}$ is taken into account, there are eight TMDs, $f_{1}\left(x, k_{T}^{2}\right), g_{1 L}\left(x, k_{T}^{2}\right)$, $h_{1}\left(x, k_{T}^{2}\right), g_{1 T}\left(x, k_{T}^{2}\right), h_{1 T}^{\perp}\left(x, k_{T}^{2}\right), h_{1 L}^{\perp}\left(x, k_{T}^{2}\right), h_{1}^{\perp}\left(x, k_{T}^{2}\right)$ and $f_{1 T}^{\perp}\left(x, k_{T}^{2}\right)$. In figure 5 . they are organised and categorised according to the quark and nucleon polarisation -unpolarised, longitudinal or transversely polarised-and also according to the quark $k_{T}$.

The TMDs can be accessed by two ways: using the Drell-Yan process [16] or SIDIS (discussed in Sect. 2). In the Drell-Yan process a quark and anti-quark annihilate into a pair of leptons, as represented in figure 6. COMPASS studies the following reaction involving an unpolarised beam $\left(H_{a}\right)$ and a transversely polarised target $\left(H_{b}\right)$ :

$$
H_{a}\left(P_{a}\right)+H_{b}\left(P_{b}, S\right) \rightarrow \gamma^{*}(q)+X \rightarrow l^{-}(l)+l^{+}\left(l^{\prime}\right)+X
$$




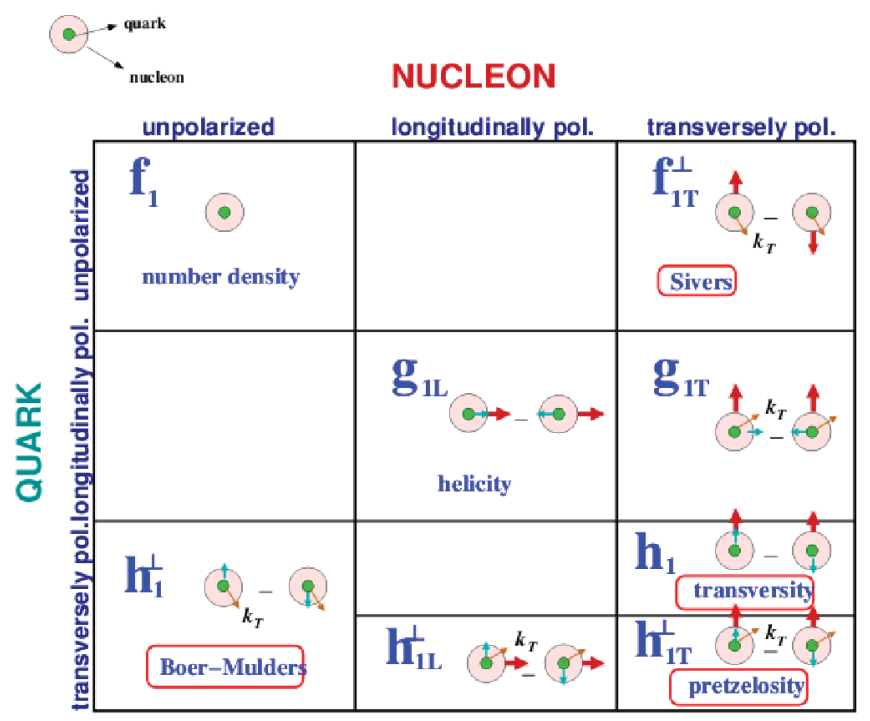

Figure 5: The eight TMDs for unpolarised beam and transversely polarised target in SIDIS.

where $P_{a(b)}$ is the momentum of the beam (target) hadron; $l, l^{\prime}$ and $q=l+l^{\prime}$ are the momenta of the lepton, the anti-lepton and the four-vector of the virtual photon, respectively, and $S$ is the four-vector of the target polarisation. Further, the following kinematical variables will be used:
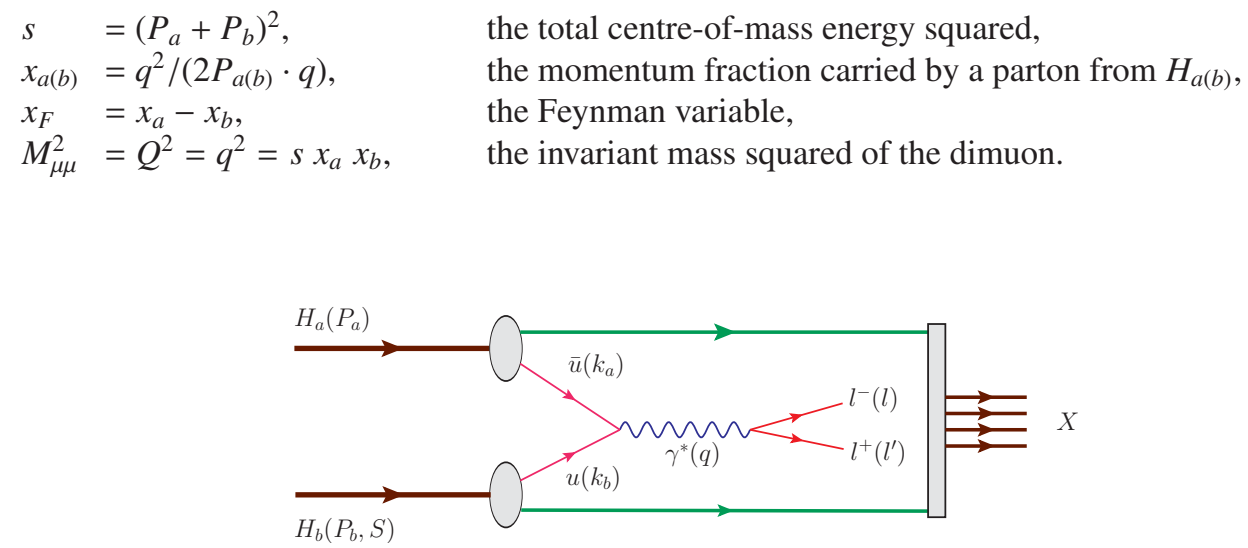

Figure 6: Schematic representation of the Drell-Yan process: the annihilation of a quark anti-quark pair into a lepton pair. 
For this special case, where the beam is unpolarised and the target is transversely polarised, in the LO QCD parton model approximation, the Drell-Yan cross section expression simplifies to

$$
\begin{aligned}
\frac{\mathrm{d} \sigma}{\mathrm{d}^{4} q \mathrm{~d} \Omega} & =\frac{\alpha_{e m}^{2}}{F q^{2}} \hat{\sigma}_{U}\left\{\left(1+D_{\left[\sin ^{2} \theta\right]} A_{U}^{\cos 2 \phi} \cos 2 \phi\right)\right. \\
& +\left|\mathbf{S}_{T}\right|\left[A_{T}^{\sin \phi_{S}} \sin \phi_{S}+D_{\left[\sin ^{2} \theta\right]}\left(A_{T}^{\sin \left(2 \phi+\phi_{S}\right)} \sin \left(2 \phi+\phi_{S}\right)\right.\right. \\
& +\frac{\left.\left.\left.A_{T}^{\sin \left(2 \phi-\phi_{S}\right)} \sin \left(2 \phi-\phi_{S}\right)\right)\right]\right\} .}{}
\end{aligned}
$$

The general form of the Drell-Yan cross section expression can be found in [17]. The solid angle $\Omega$ specifies the orientation of the lepton, $F=4 \sqrt{\left(P_{a} \cdot P_{b}\right)^{2}-M_{a}^{2} M_{b}^{2}}$ represents the flux of incoming hadrons. The angles $\phi$ and $\theta$ are defined in the Collins-Soper frame, while the angle $\phi_{S}$ is defined in the target rest frame. The part of the cross section $\hat{\sigma}_{U}=F_{U}^{1}\left(1+\cos ^{2} \theta\right)$ is factored out, after integration over the azimuthal angles $\phi$ and $\phi_{S}$, where $F_{U}^{1}$ is the unpolarised structure function $F_{1}$. The depolarisation factor $D_{[f(\theta)]}$ depends only on the lepton polar angle $\theta$, as $D_{[f(\theta)]}=f(\theta) /\left(1+\cos ^{2} \theta\right)$.

The four azimuthal asymmetries, $A_{P}^{f\left(\phi, \phi_{S}\right)}$, in equation (5), are obtained by the ratios of the combinations of structure functions $F_{P}^{f\left(\phi, \phi_{S}\right)}$ over $F_{U}^{1}+F_{U}^{2}$. In this particular case $P$ stands for $U$ or $T$ for the unpolarised and for the transversely polarised case, respectively. More precisely these azimuthal asymmetries:

- $A_{U}^{\cos 2 \phi}$ gives access to the Boer-Mulders functions of the incoming hadrons and target nucleon,

- $A_{T}^{\sin \phi_{S}}$ to the Sivers function of the target nucleon,

- $A_{T}^{\sin \left(2 \phi+\phi_{S}\right)}$ to the Boer-Mulders function of the beam hadron and to $h_{1 T}^{\perp}$, the pretzelosity function of the target nucleon,

- $A_{T}^{\sin \left(2 \phi-\phi_{S}\right)}$ to the Boer-Mulders function of the beam hadron and $h_{1}$, the transversity function of the target nucleon.

Figure 7. shows the projected statistical errors for the azimuthal asymmetries, as a function of $x-$ Feynman, of the Drell-Yan measurement $\pi^{-} p \rightarrow \mu^{+} \mu^{-} X$ with a $190 \mathrm{GeV} / c$ pion beam, in the so-called high mass region, $4 \mathrm{GeV} / c^{2}<M_{\mu \mu}<9 \mathrm{GeV} / c^{2}$. For Boer-Mulders the statistical error is spread into 5 bins, while for the other three only one bin is used. These errors were calculated considering a 140 days run at the beam intensity of $10^{8}$ pions/s. Thus, the rate of Drell-Yan events in the high mass region expected per day is 2034, in total 285000 Drell-Yan events are expected in the mass region 4 $9 \mathrm{GeV} / c^{2}$. More information about the calculation of this estimation can be found on Ref. [18].

In particular, within the context of this new program of the COMPASS experiment, the Sivers and the Boer-Mulders functions can be extracted from Drell-Yan processes and SIDIS events, as these functions are $T$-odd, i.e. both functions are process dependent, therefore the following relations should hold:

$$
\left.f_{1 T}^{\perp}\right|_{D Y}=-\left.f_{1 T}^{\perp}\right|_{D I S} \quad \text { and }\left.\quad h_{1}^{\perp}\right|_{D Y}=-\left.h_{1}^{\perp}\right|_{D I S},
$$

This represents a crucial test of non-perturbative QCD and of the TMD approach that COMPASS has proposed to perform.

\section{Acknowledgements}

This work was partially funded by Fundação para a Ciência e Tecnologia. 

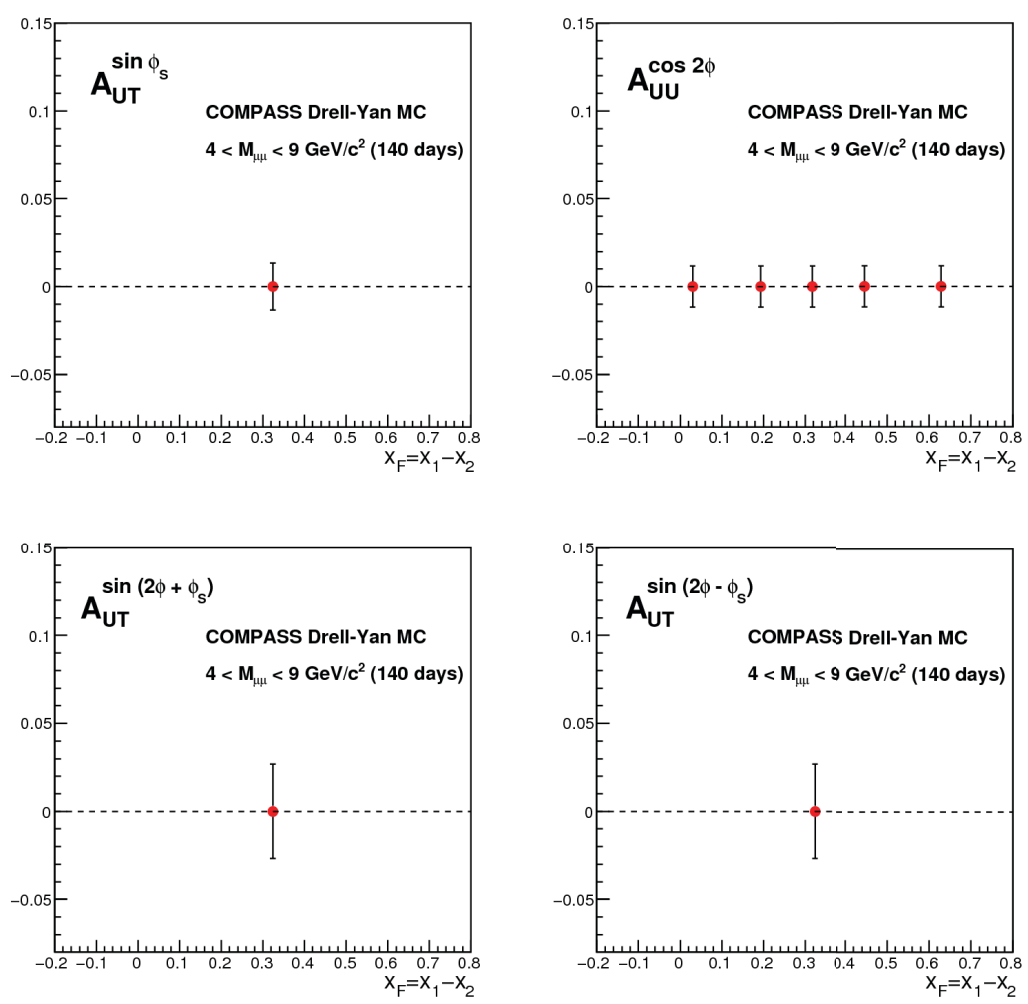

Figure 7: The expected predictions on the statistical errors for the Sivers (top-left), Boer-Mulders (top-right), $\sin \left(2 \phi+\phi_{S}\right)$ (bottom-left) and $\sin \left(2 \phi-\phi_{S}\right)$ (bottom-right) azimuthal asymmetries for a DY measurement $\pi^{-} p \rightarrow \mu^{+} \mu^{-} X$ with a $190 \mathrm{GeV} / c \pi^{-}$beam in the high-mass region.

\section{References}

[1] P. Abbon et al. [COMPASS Collaboration], Nucl. Instrum. Meth. A577, 455 (2007)

[2] F. Gautheron et al. [COMPASS Collaboration], CERN-SPSC, 014 (2010)

[3] C. Adolph [COMPASS Collaboration] et al., Phys. Lett. B736, 124 (2014)

[4] C. Adolph [COMPASS Collaboration] et al., Eur. Phys. J. 73, 2581 (2014)

[5] C. Adolph [COMPASS Collaboration] et al., Phys. Lett. B717, 376 (2012); Phys. Lett. B717, 383 (2012)

[6] A. Airapetian et al. [HERMES Collaboration], Phys. Lett. B666, 446 (2008)

[7] A. Airapetian et al. [HERMES Collaboration], Phys. Rev. D89, 097101 (2014)

[8] A.D. Martin, W.J. Stirling, R.S. Thorne, G. Watt, Eur. Phys. J. C63, 189 (2009)

[9] X.-D. Ji, Phys. Rev. Lett. 78, 610 (1997)

[10] M. Burkardt, Phys. Rev. D62, 071503 (2000), Erratum ibid. D66, 119903 (2002)

[11] M. Diehl, Eur. Phys. J. C25, 223 (2002)

[12] M. Burkardt, arXiv:0711.1881 
[13] F. D. Aaron et al. [H1 Collaboration], Phys. Lett. B 659, 796 (2008)

[14] A. Aktas et al. [H1 Collaboration], Eur. Phys. J. C44, 1 (2005)

[15] S. Chekanov et al. [ZEUS Collaboration], JHEP 05, 108 (2009)

[16] S.D. Drell, T.M. Yan, Phys. Rev. Lett. 25, Erratum-ibid. 25, 902 (1970)

[17] S. Arnold, A. Metz, M. Schlegel, Phys. Rev. D79, 034005 (2009)

[18] M. Quaresma, et al., COMPASS note 2014-11 (2014) 